\title{
Congenital heart disease affects local gyrification in 22q11.2 deletion syndrome
}

\author{
MARIE SCHAER MD PHD ${ }^{1,2}$ | BRONWYN GLASER MA ${ }^{1}$ | MERITXELL BACH CUADRA PHD ${ }^{2}$ | \\ MARTIN DEBBANE PHD ${ }^{1}$ | JEAN-PHILIPPE THIRAN PHD ${ }^{2}$ | STEPHAN ELIEZ MD ${ }^{1,3}$
}

1 Service Médico-Pédagogique, Department of Psychiatry, University of Geneva School of Medicine, Geneva, Switzerland. 2 Signal Processing Institute, Swiss Federal Institute of Technology, Lausanne, Switzerland. 3 Department of Genetic Medicine and Development, University of Geneva School of Medicine, Geneva, Switzerland.

Correspondence to Dr Marie Schaer, Service Médico-Pédagogique, 1 rue David Dufour, Case Postale 50, 1211 Geneva 8, Switzerland.

E-mail: marie.schaer@unige.ch

\section{PUBLICATION DATA}

Accepted for publication 3rd November 2008.

Published online 11th March 2009

\section{LIST OF ABBREVIATIONS}

22q11.2DS 22q11.2 deletion syndrome

CHD Congenital heart disease

FSI0 Full-scale I0

GI Local gyrification index

PIO Performance IO

VIO Verbal I0

\section{ACKNOWLEDGMENTS}

This research was supported by Swiss National Research Funds (grant 323500111165 to MS and grants 3200-063135, 3232063134, and PP00B-102864 to SE) and by NARSAD Institute, the Center for Biomedical Imaging of the Geneva - Lausanne Universities, the EPFL, as well as the Leenaards and Louis-Jeantet foundations (http:// www.cibm.ch).
$22 q 11.2$ deletion syndrome (22q11.2DS) is a common genetic condition associated with cognitive and learning impairments. In this study, we applied a threedimensional method for quantifying gyrification at thousands of points over the cortical surface to imaging data from 44 children, adolescents, and young adults with 22q11.2DS ( 17 males, 27 females; mean age 17y $2 \mathrm{mo}$ [SD 9y $1 \mathrm{mo}$ ], range 6-37y), and 53 healthy participants ( 21 males, 32 females; mean age $15 \mathrm{y} 4 \mathrm{mo}$ [SD 8 y $6 \mathrm{mo}$ ]; range 6-40y). Several clusters of reduced gyrification were observed, further substantiating the pattern of cerebral alterations presented by children with the syndrome. Comparisons within 22q11.2DS demonstrated an effect of congenital heart disease (CHD) on cortical gyrification, with reduced gyrification at the parieto-temporo-occipital junction in patients with CHD, as compared with patients without CHD. Reductions in gyrification can resemble mild polymicrogyria, suggesting early abnormal neuronal proliferation or migration and providing support for an effect of hemodynamic factors on brain development in 22q11.2DS. The results also shed light on the pathophysiology of acquired brain injury in other populations with CHD.
$22 \mathrm{q} 11.2$ deletion syndrome (22q11.2DS) is a neurogenetic disorder affecting 1 in 5000 live births. ${ }^{1}$ Children with 22q11.2DS typically present with impairment in domains such as memory and attention, as well as fine motor skills and visuospatial abilities. ${ }^{2}$ Previous attempts at delineating the cortical correlates responsible for cognitive deficits in children and adolescents with the 22q11.2DS have consistently identified reductions in cortical grey matter, ${ }^{2-4}$ detecting some of the regions that are most altered in the syndrome (e.g. the parietal lobe). However, volumetric alterations are a product of changes to either cortical thickness or area, the latter of which is dependent on the degree of folding. Therefore, new three-dimensional methods that measure thickness and gyrification may contribute more detailed information on the etiopathogenesis of cortical alterations.

While cortical thickness is particularly informative about the dynamics of cortical maturation, ${ }^{5}$ changes in cortical morphology may help to elucidate early developmental processes. For example, enlarged Sylvian fissures in children with 22q11.2DS can result from abnormal opercular development during embryogenesis. ${ }^{6}$ In a previous study using a two-dimensional method, we identified a reduced gyrification index in the frontal and parietal lobes, ${ }^{7}$ suggesting a simplified gyral pattern and decreased surface area in these regions. However, two-dimensional methods are not optimal for quantifying complex cortical convolutions. More recently, we tested a new three-dimensional 
technique for the quantification and localization of cortical surface changes, ${ }^{8}$ improving both accuracy and overall precision.

Understanding the etiopathogenesis of cortical anomalies in 22q11.2DS also relies on the identification of factors that determine phenotypic expression. Congenital heart disease (CHD), which affects more than half of individuals with 22q11.2DS, ${ }^{9}$ may be one such factor because it is known to alter brain structure and affect neurodevelopmental outcomes in non-syndromic children. ${ }^{10,11}$ Previous studies of 22q11.2DS have not demonstrated an impact of cardiac defects on cerebral phenotype., ${ }^{4,6,12}$ Our inability to differentiate between patients with 22q11.2DS, with and without CHD, however, may be due to methodological problems such as small sample sizes and the grouping of participants with widely varying CHD. Moreover, volumetric studies can easily miss cortical changes in certain regions. To address these issues, the present study included the following: (1) one of the largest samples of children, adolescents, and young adults with the syndrome collected to date; (2) patients with 22q11.2DS with and without CHD based on strict cardiac surgery criteria; and (3) a newly developed method, independent of regional a priori hypotheses and boundaries, to determine the extent to which CHD affects the expression of gyral anomalies in 22q11.2DS.

\section{METHOD}

\section{Participants}

Forty-four children, adolescents and young adults with 22q11.2DS aged 6 to 37 years (17 males, 27 females; mean age $17 \mathrm{y} 2 \mathrm{mo}$ [SD $9 \mathrm{y} 1 \mathrm{mo}]$ ) were recruited through announcements to regional parent associations in Switzerland and France. 22q11.2DS was confirmed in all participants. Mean Full-scale IQ (FSIQ) in the 22q11.2DS group was 68.5 (SD 12.1).

The comparison group comprised of 53 typically developing individuals (21 males, 32 females) aged 6 to 40 years (mean age $15 \mathrm{y} 4 \mathrm{mo}$ [SD $8 \mathrm{y} 6 \mathrm{mo}$ ) was recruited through a newsletter distributed at public schools and in the Geneva community. A medical interview, as well as parent-report Child Behavior Checklist ${ }^{13}$ in children and adolescents) and self-report (Symptoms Checklists Youth Self-Report Form ${ }^{14}$ and Auto-Adult Behaviour Checklist $(\mathrm{ABCL})^{15}$ in adolescents and adults) behavioral questionnaires were used to screen comparison participants. Mean FSIQ in the comparison group was 111.9 [SD 13.2]. Individuals with a history of past or present neurological or psychiatric disorders were excluded. Written informed consent was received from all participants, as well as from the parents of participants younger than 18 years of age, in accordance with protocols approved by the
Institutional Review Board of Geneva University School of Medicine.

\section{CHD in 22q11.2DS}

Parents of participants were asked if their child had signs of a cardiac problem or had undergone heart surgery. In addition, medical reports, including ultrasound examinations and surgical reports, were examined. Seventeen of the 44 patients with 22q11.2DS (seven males, 10 females; mean age 14y 6mo [SD 8y $1 \mathrm{mo}]$ ) were identified as having a history of major CHD (six persons with tetralogy of Fallot, four with interrupted aortic arch, and seven with various septal defects commonly associated with pulmonary hypertension). All patients in the CHD subgroup had undergone palliative or reparative surgery before the age of 4 years, except one 6-year-old female who was to be operated on shortly after participation. Nine other patients were excluded from CHD analyses (mean age 17y [SD 6]), due to unclear cardiovascular status (including one patient with retro-oesophagal subclavian artery and five patients with a history of ventricular septal defect with spontaneous closure). There were 18 patients in the subgroup without CHD (seven males, 11 females; mean age $20 \mathrm{y}$ [SD $10 \mathrm{y} 8 \mathrm{mo}$ ), all with normal cardiac ultrasounds and with a history of cardiorespiratory complications at birth. Although the age difference between the CHD and no-CHD subgroups did not reach statistical significance $(p=0.134)$, we controlled for age in all statistical comparisons.

FSIQ was similar in the subgroup with CHD (67.8 [SD 12.9]) and without CHD (67.4 [SD 12.1]), but there was a non-significant trend toward lower IQ in the six patients with tetralogy of Fallot (FSIQ 62.8 [SD 12.4]; age, 14.1 [SD 8.4]), as compared with the 11 with acyanotic CHD (interrupted aortic arch and septal defects combined; FSIQ 70.5 [SD 12.8]; age, 14.7 [SD 8.3]). Given non-significant age differences between the CHD and no-CHD groups, we subsequently divided children, adolescents (younger than 16y), and adults to check for age-related IQ differences in the CHD groups. Exploratory IQ comparisons in patients younger than 16 years of age did not demonstrate significant differences in FSIQ, Verbal IQ (VIQ), or Performance IQ (PIQ) between children with and without CHD. However, the VIQ>PIQ dissociation frequently observed in 22q11.2DS ${ }^{16}$ was seen in children with CHD. The 11 children with CHD scored a mean of 10 points higher on VIQ than PIQ, whereas the nine children without CHD scored 4 points lower on VIQ than PIQ (twosample Student's $t$-test: $p=0.021, t(18)=-2.879)$. In patients older than 16 years, this IQ dissociation was no longer apparent, but adults with CHD had significantly lower 
VIQ ( $n=6$, VIQ 55 [SD 2.3]) than adults without ( $n=9$; VIQ 69.8 [SD 13.7]; two-sample Student's $t$-test: $p=0.026$, $t(13)=2.506)$.

\section{Magnetic resonance image acquisition and processing}

Cerebral magnetic resonance images were acquired with a three-dimensional volumetric pulse sequence using a Philips Intera 1.5T scanner (Phillips Healthcare, Best, the Netherlands) The following scan parameters were used: $\mathrm{TR}=35 \mathrm{msec}, \mathrm{TE}=6 \mathrm{msec}$, flip angle $=45^{\circ}, \mathrm{NEX}=1$, matrix size $=256 \times 192$, field of view $=24 \mathrm{~cm}^{2}$, slice thickness $=1.5 \mathrm{~mm}, 124$ slices. Images were first imported into FreeSurfer software (https://surfer.nmr.mgh.harvard.edu/) to produce precise three-dimensional representations of the cortical surfaces. A detailed description of image analysis is provided by the developers. ${ }^{17}$ The white and pial surfaces were then carefully inspected, and topological defects contributing to surface inaccuracies were manually removed.

Based on the pial surface reconstruction produced in FreeSurfer, we developed an algorithm for measuring three-dimensional local gyrification index (lGI) at thousands of points across each hemisphere. This method, denoted as lGI, is now fully implemented in FreeSurfer, and thus freely available to the scientific community. Details of the $l$ GI computation can be found in our validation paper $^{8}$ and at https://surfer.nmr.mgh.harvard.edu/ fswiki/LGI; a brief description is presented below.

The $l$ GI method is adapted from the classical gyrification index $\left(2 \mathrm{D}-\mathrm{GI}^{18}\right)$, which is the ratio of the total pial cortical surface over the perimeter of the brain delineated on coronal sections. However, 2D-GI can present threats to accuracy, given that coronal slices do not take into account the inherent three-dimensional nature of the cortical surface. More specifically, perimeter measurements can easily become biased by slice orientation, or by the presence of buried sulci. Also, the use of two-dimensional slices does not allow for precise localization of gyral anomalies. Our method, lGI, addresses these issues by iteratively quantifying GI in circular three-dimensional regions of interest. After the creation of an outer envelope that tightly wraps the pial cortical surface, local measurement of circular GI is computed for each vertex of the outer surface as the ratio of corresponding regions of interest (ROI) on the hull and pial meshes. Delineation of the ROI on both the outer surface $\left(\mathrm{ROI}_{O}\right)$ and pial surface $\left(\mathrm{ROI}_{P}\right)$ uses a matching algorithm based on geodesic constraints, so that the $\mathrm{ROI}_{P}$ takes into account the entire patch of the cortical surface delineated by the $\mathrm{ROI}_{O}$ circular perimeter (see Fig. 1). This means that at the end of the computational process, individual $l$ GI cortical maps reflect the amount of cortex buried within the sulcal folds in the surrounding circular region (see Fig. 1, Panel C).

\section{Statistical analyses}

After the steps described above, FreeSurfer software was used to conduct vertex-based statistical analyses. A studyspecific template was created by averaging the surfaces of the 97 participants. Individual cortical surfaces were then registered to the canonical surface, optimally aligning sulcal features across participants, and data were resampled to a common average spherical coordinate system. To increase the signal-to-noise ratio, resampled cortical maps of $l$ GI values were smoothed, using an iterative nearestneighbor averaging procedure, with the full width at a half maximum of $10 \mathrm{~mm}$.

Statistical $l$ GI difference maps were created by computing a general linear model (GLM) to assess the effect of diagnosis on the $l$ GI value at each vertex. Sex was entered as a potential confounding factor in the GLM, and age effects were controlled for by modeling the slope of $l$ GI regressed on age in each subgroup ('different offset - different slope design'). Corrections for multiple comparisons were performed using a false discovery rate (FDR) of 0.01 to set the significance threshold. ${ }^{19}$ To complement the vertex-wise maps of statistical differences, the GLM allowed for the extraction of mean $l$ GI values for each cluster demonstrating significant differences; series of analyses of covariance (ANCOVA) were then conducted on mean $l$ GI per cluster, using age and sex as covariates. These confirmatory ANCOVA did not model a different effect of age on $l$ GI in each subgroup (i.e. GLM with 'same slope design').

A second GLM was then performed at each vertex to assess the effect of CHD on $l$ GI distribution in the CHD versus no-CHD 22q11.2DS subgroups, using age and sex as potential confounding factors. Similarly to the patients versus the comparison group analysis, mean $l$ GI values in clusters of significant differences between the CHD and no-CHD subgroups were extracted and confirmed using series of ANCOVA.

\section{RESULTS}

\section{Patients versus comparison group}

Mean total cerebral volume was significantly smaller in patients $\left(937.7 \mathrm{~cm}^{3}\right.$ [SD 112.7]) compared with the comparison group (1062.3 $\mathrm{cm}^{3}$ [SD 106.0]; $F=31.4, p<0.001$ ). Table I shows a significant reduction in mean $l$ GI in the patient compared with the comparison group. Within both the patient and comparison groups, $l$ GI decreased significantly with age (all $p<0.001$, comparison: left hemisphere, $\mathrm{R}=-0.574$, right hemisphere, $\mathrm{R}=-0.631$; patients: left, 

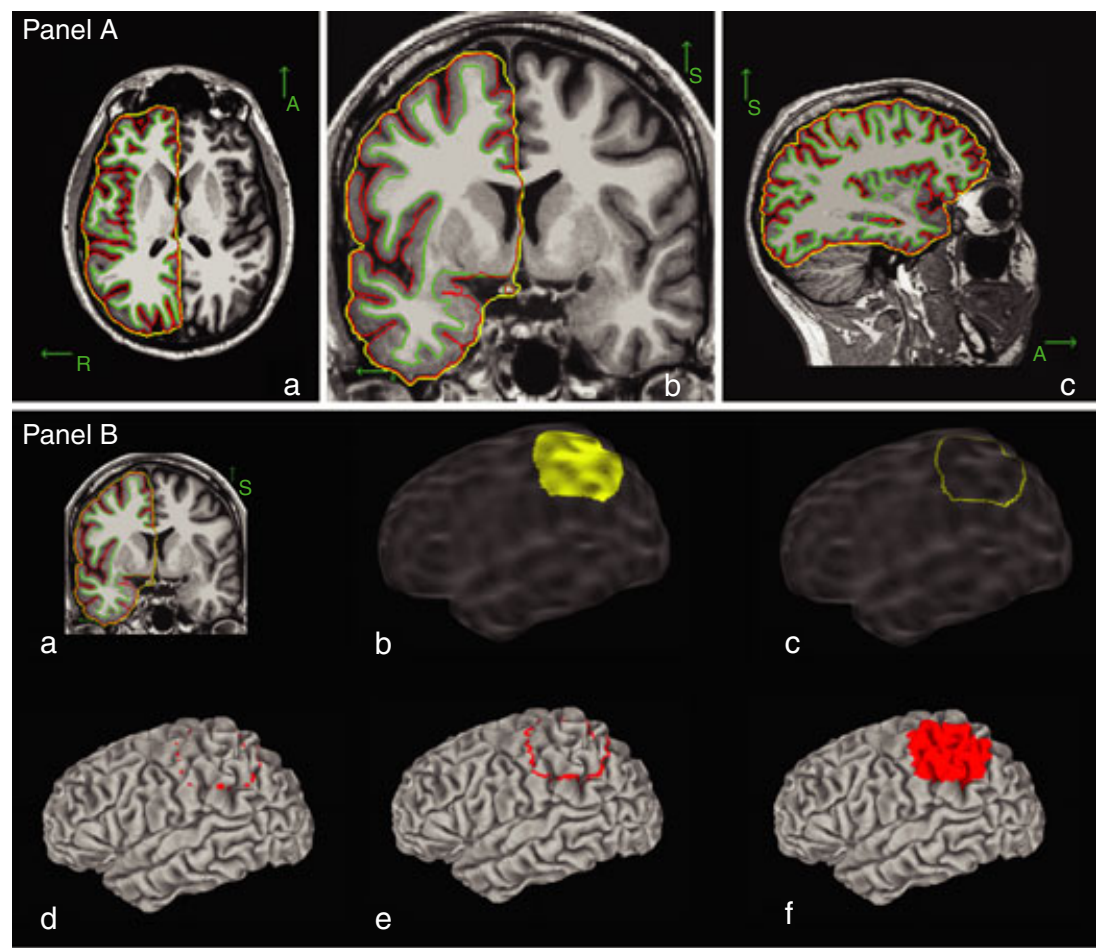

d

e
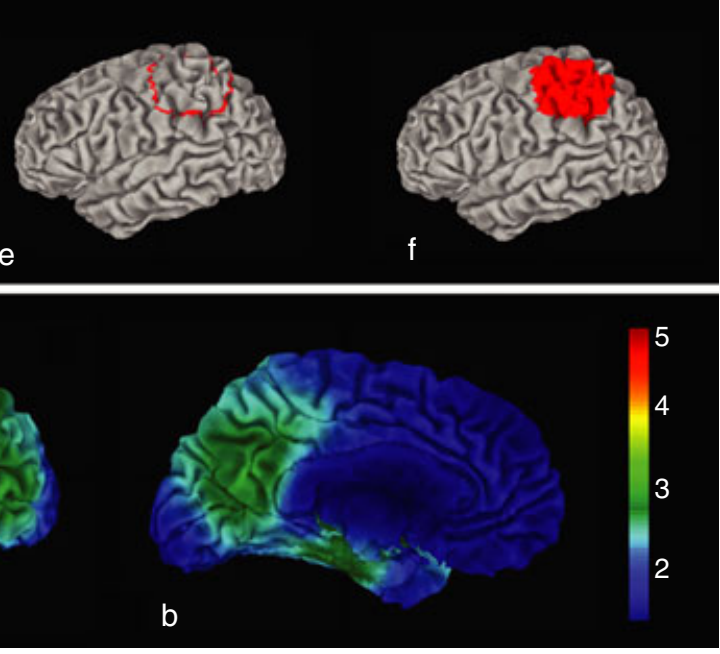

\begin{abstract}
Figure 1: Local gyrification index (/GI) computation. In Panel A, the white matter (in green) and pial (in red) cortical surfaces obtained using FreeSurfer software are overlaid on (a) axial, (b) coronal, and (c) sagittal magnetic resonance images. The outer surface wrapping the brain is shown in yellow. Panel B shows mapping of a circular region of interest on the outer surface ( $\mathrm{ROI}_{O}$, in yellow) to a corresponding region of interest on the pial surface ( $\left(\mathrm{ROI}_{B}\right.$ in red). This correspondence is achieved through the transfer of a set of points from the $\mathrm{ROI}_{O}$ perimeter to the pial surface, which is then used to reconstruct the full geodesical cortical $\mathrm{ROI}_{P}$. Panel $\mathrm{C}$ shows an example of an /GI-overlaid cortical map for a control participant on (a) lateral and (b) medical views.
\end{abstract}

$\mathrm{R}=-0.517$, right, $\mathrm{R}=-0.510$ ), and was significantly greater in males than females (comparison: left, $F=7.9, p=0.007$, right, $F=9.8, p=0.003$; patients: left, $F=8.3, p=0.006$, right, $F=4.3, \quad p=0.045)$. These between-group differences remained robustly significant when covarying for age and sex (left, $p<0.001 ; F_{3,93}=49.0$, right, 72.2 ), and when covarying for age, sex, and total brain volume $(p<0.001$; left, $F_{4,92}=17.4$, right, 30.3).

Cortical maps illustrate clusters of significant reduction in the patient and comparison groups, after controlling for the effects of sex and age at each vertex (Fig. 2, left panel). Mean $l$ GI values in significant clusters, along with the $F$-tests details, are presented in Table I.

\section{Effect of CHD}

Total cerebral volume was significantly smaller in patients with CHD (889.4 $\mathrm{cm}^{3}$ [SD 124.0]) compared with the noCHD group (979.6 $\mathrm{cm}^{3}$ [SD 94.4]; $F=5.9, p=0.021$ ). This reduction remained highly significant when covarying for age and sex $\left(F_{3,31}=11.3, p=0.002\right)$. Subsequent analysis of the tissular compartments revealed that the reduction equally affected grey $\left(F_{3,31}=9.3, p=0.005\right)$ and white matter $\left(F_{3,31}=7.6, p=0.010\right)$.

Comparisons of mean $l$ GI values revealed a reduction in the right hemisphere of patients with CHD versus patients without $\mathrm{CHD}(F=4.7, p=0.038)$ and a trend toward reduction in the left hemisphere $(F=3.6$, 
Table I: Mean local gyrification index (/GI) values for clusters demonstrating significant reduction in patients with 22q11.2DS compared with the non-affected group

\begin{tabular}{|c|c|c|c|c|}
\hline & $\begin{array}{l}\text { Area } \\
\left(\mathrm{mm}^{2}\right)\end{array}$ & Comparison & Patients & $F$ \\
\hline Mean Right /GI & & $2.481(0.120)$ & $2.322(0.092)$ & $51.2^{\mathrm{a}}$ \\
\hline $\begin{array}{l}\text { Right pre- and post-central gyri, extending into the } \\
\text { supramarginal gyrus and PTO junction }\end{array}$ & 7763.7 & $2.945(0.169)$ & $2.728(0.119)$ & $71.835^{\mathrm{a}}$ \\
\hline Right posterior cingulate gyrus & 6217.8 & $2.313(0.173)$ & $1.994(0.125)$ & $133.990^{\mathrm{a}}$ \\
\hline Right orbitofrontal cortex & 1972.8 & $1.644(0.078)$ & $1.544(0.784)$ & $39.252^{\mathrm{a}}$ \\
\hline Right superior temporal gyrus (anterior part) & 1035.7 & $3.323(0.273)$ & $3.089(0.234)$ & $20.548^{\mathrm{a}}$ \\
\hline Right middle frontal gyrus & 572.4 & $2.709(0.201)$ & $2.461(0.175)$ & $45.841^{\mathrm{a}}$ \\
\hline Mean Left IGI & & $2.475(0.134)$ & $2.319(0.106)$ & $38.9^{\mathrm{a}}$ \\
\hline Left posterior cingulate gyrus extending into the occipital pole & 7921.3 & $2.463(0.231)$ & $2.151(0.141)$ & $68.439^{\mathrm{a}}$ \\
\hline $\begin{array}{l}\text { Left pre- and post-central gyrus extending into the superior } \\
\text { frontal gyrus }\end{array}$ & 6626.5 & $2.854(0.218)$ & $2.662(0.145)$ & $27.214^{\mathrm{a}}$ \\
\hline Left PTO junction & 1229.7 & $2.828(0.193)$ & $2.637(0.131)$ & $29.800^{\mathrm{a}}$ \\
\hline Left medial frontal gyrus (rostral part) & 1175.8 & $1.620(0.082)$ & $1.522(0.992)$ & $30.520^{\mathrm{a}}$ \\
\hline Left middle frontal gyrus & 840.4 & $2.485(0.199)$ & $2.268(0.159)$ & $33.594^{\mathrm{a}}$ \\
\hline Left orbitofrontal cortex & 594.9 & $2.107(0.194)$ & $1.960(0.154)$ & $15.475^{\mathrm{a}}$ \\
\hline
\end{tabular}

${ }^{\mathrm{a}} p<0.001$. PTO, parieto-temporo-occipital.

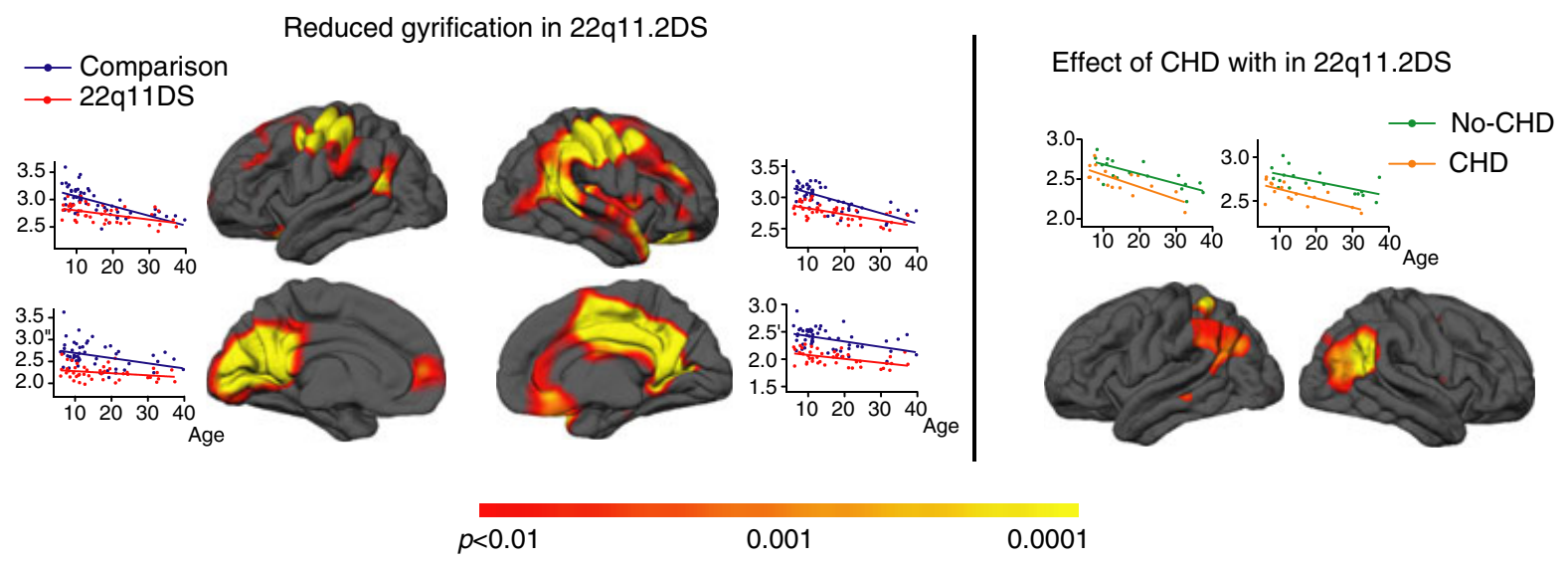

Figure 2: Statistical maps of the vertex-by-vertex local gyrification index (/GI) comparisons (colorbar shows $p$ values). Left panel shows regions of decreased IGI in patients with 22q11.2DS compared with the comparison groups (FDR<0.01). No region of increased /GI was observed in 22q11.2DS compared with comparison groups. A detailed description of the clusters is provided in Table I. The right panel illustrates reduced /GI in the congential heart disease (CHD) compared to no-CHD subgroups $(p<0.01)$. The cluster at the right parieto-temporo-occipital junction was more significant than the left one, and survived correction for multiple comparisons using FDR $<0.05$. No cluster of increased IGI value was observed in the subgroup with CHD compared with the subgroup without. Details of the mean IGI values per cluster are available in Table II. FDR, false discovery rate.

$p=0.069$ ), when covarying for age and sex. Vertex-wise analyses showed clusters of decreased $l$ GI in the CHD group at the parieto-temporo-occipital junction bilaterally, as well as smaller parietal clusters (Fig. 2, right panel). Mean $l$ GI values for the corresponding clusters are presented in Table II.

To identify whether gyral anomalies were associated with alterations to cortical thickness, we further explored differences in cortical thickness between patients with and without CHD using two different methods. First, using vertex-by-vertex whole-brain statistical analyses, we did not observe regional differences in cortical thickness between the two subgroups ( $p<0.05$, uncorrected). Second, we did not observe changes in cortical thickness associated with CHD in the four regions corresponding to differences in $l$ GI (see Table II). 
Table II: Mean local gyrification index (IGI) and cortical thickness values for clusters demonstrating significant /GI differences between subgroups of patients with 22q11.2DS, with and without congenital heart disease (CHD)

\begin{tabular}{|c|c|c|c|c|c|c|c|}
\hline & \multirow[b]{2}{*}{ Area $\left(\mathrm{mm}^{2}\right)$} & \multicolumn{3}{|c|}{ IGI } & \multicolumn{3}{|c|}{ Cortical thickness } \\
\hline & & $\begin{array}{l}\text { Patients } \\
\text { without } \\
\text { CHD (n=18) }\end{array}$ & $\begin{array}{l}\text { Patients } \\
\text { with CHD } \\
(n=17)\end{array}$ & $\begin{array}{l}\text { Excluded } \\
\text { subgroup } \\
(n=9)\end{array}$ & $\begin{array}{l}\text { Patients } \\
\text { without } \\
\text { CHD }(n=18)\end{array}$ & $\begin{array}{l}\text { Patients } \\
\text { with } \\
\mathrm{CHD}(n=17)\end{array}$ & $\begin{array}{l}\text { Excluded } \\
\text { subgroup } \\
(n=9)\end{array}$ \\
\hline Left PTO junction & 3448.2 & $2.716(0.107)^{\mathrm{a}}$ & $2.602(0.151)^{a}$ & $2.664(0.126)$ & $2.674(0.337)$ & $2.800(0.297)$ & $2.700(0.224)$ \\
\hline Left parietal superior & 364.6 & $2.585(0.175)^{\mathrm{b}}$ & $2.492(0.165)^{\mathrm{b}}$ & $2.497(0.195)$ & $2.317(0.219)$ & $2.220(0.407)$ & $2.089(0.392)$ \\
\hline Right PTO junction & 3702.7 & $2.689(0.136)^{\mathrm{c}}$ & $2.565(0.121)^{\mathrm{c}}$ & $2.638(0.124)$ & $2.596(0.356)$ & $2.732(0.331)$ & $2.606(0.235)$ \\
\hline Right parieto-occipital & 366.1 & $2.430(0.160)^{\mathrm{d}}$ & $2.361(0.135)^{d}$ & $2.358(0.163)$ & $2.167(0.278)$ & $2.258(0.232)$ & $2.330(0.298)$ \\
\hline
\end{tabular}

Values for the subgroup of patients with unclear cardiac status are shown for information only, these individuals were not included in the statistical analyses. According to the analyses of covariance, the $/ \mathrm{GI}$ differences were significant at $p<0.001$ with the values of ${ }^{\mathrm{a}} F=16.231$; ${ }^{\mathrm{b}} F=15.769 ;{ }^{\mathrm{c}} \mathrm{F}=27.905 ;{ }^{\mathrm{d}} \mathrm{F}=13.995$. To test whether differences in $/ \mathrm{Gl}$ were associated with change to cortical thickness, multiple analyses of covariance (MANCOVA) were conducted to compare mean cortical thickness values per cluster between CHD and no-CHD, using age and sex as covariates. While IGI differences between the CHD and no-CHD subgroups were found to be highly significant, MANCovA comparing mean cortical thickness values for each cluster demonstrated no difference in thickness between subgroups (Wilks' Lambda: $F_{4,28}=1.084$, $p=0.384$ ). PTO, parieto-temporo-occipital.

Finally, exploratory statistics comparing local gyrification between the six patients with tetralogy of Fallot and the 11 with acyanotic CHD did not reveal differences in gyral anomalies using an alpha level of $p<0.05$ (uncorrected). Larger samples may be needed to determine the effects of specific cardiac malformations on gyral anomalies.

\section{DISCUSSION}

Using a method unrestricted by lobar boundaries, we observed several clusters of reduced local gyrification in 22q11.2DS. Given that the cortex grows through radial expansion, ${ }^{20}$ decreased surface area in circular ROI supports an early defect of cortical expansion. This reduced surface area is likely to be additionally expressed in reduced grey matter quantity in the altered cortical regions. Indeed, bilateral $l$ GI reduction in lateral (inferior parietal cortex, parieto-temporo-occipital junction, middle frontal gyrus) and medial (posterior cingulate gyrus) regions is consistent with previous volumetric studies in the syndrome..$^{2-4}$

\section{Previous findings in 22q11.2DS}

Consistent with our $l$ GI findings, reduced cortical volumes in the inferior parietal lobe and supramarginal gyrus have been observed in previous studies of children and adolescents with 22q11.2DS. ${ }^{2-4}$ In addition to structural anomalies, aberrant functional activation was also reported in these areas, such as increased activation during arithmetic reasoning in $22 \mathrm{q} 11.2 \mathrm{DS}^{21}$ Cortical changes in the inferior parietal region are also thought to affect spatial reasoning, ${ }^{2}$ as well as complex cognitive manipulations relying on fronto-parietal connections, like working memory. ${ }^{22}$

Our findings also point to gyrification changes in regions less frequently discussed in 22q11.2DS, such as the cingulate gyrus. The first study that analyzed cortical changes on the medial aspect of the brain, using a method capable of detecting regional differences with (sublobar) precision, showed decreased grey matter density in the cingulate gyrus. ${ }^{2}$ The authors suggested that reduced cingulate volume may contribute to frequently cited attentional deficits in $22 \mathrm{q} 11.2 \mathrm{DS},{ }^{23}$ as well as impairments to patients' 'sense of self', an awareness particularly relevant to psychotic manifestations frequently experienced by affected individuals. ${ }^{2}$ More recently, we confirmed cingulate reduction using a ROI method, and further observed a significant relationship between volumetric reduction and the occurrence of psychotic symptoms in $22 \mathrm{q} 11.2 \mathrm{DS} .{ }^{24}$ The results presented here show reduced cortical surface area in the cingulate region, suggesting that early abnormalities in neuronal migration affecting gyrification may contribute to volumetric reductions. These cingulate reductions may, in turn, predispose individuals with 22q11.2DS to develop psychosis.

Pre- and post-central cortical areas have received less attention in 22q11.2DS, despite the fact that analogous alterations to white matter tracts have been reported. ${ }^{22}$ We observed reduced gyrification in the primary motor and somatosensorial areas, regions largely implicated in hand and facial movements. A decreased $l \mathrm{GI}$ in those primary cortical areas supports impairments in fine motor skills (manual dexterity and grapho-motor control) fre- 
quently reported in the syndrome. ${ }^{25}$ Similarly, oromotor control, related to the language difficulties typically associated with $22 \mathrm{q} 11.2 \mathrm{DS},{ }^{5}$ may also be affected by the cortical anomalies.

\section{Underlying etiopathogenesis}

Defects in cortical expansion, as demonstrated by $l \mathrm{GI}$ reduction in 22q11.2DS, point toward early abnormalities in control over neuronal migration or proliferation. It would be tempting to think of medial changes in $l \mathrm{GI}$ as yet another midline anomaly resulting from genetically induced disturbance to neural crest development. ${ }^{26}$ However, abnormal control over neural crest development can only partially explain the pattern of $l$ GI findings reported here. Cases of polymicrogyria, a severe cortical malformation frequently associated with $22 \mathrm{q} 11.2 \mathrm{DS},{ }^{27}$ may shed light on alternative explanations for the observed cortical anomalies. Polymicrogyria is often traced back to ischemia, ${ }^{28}$ implicating cardio-vascular defects in gyrification changes. Given that polymicrogyria appears on magnetic resonance imaging as extreme surface reduction due to sulcal shallowing, the degree of the reported $l$ GI reductions in 22q11.2DS seems to qualify as subtle cortical deformation in the direction of polymicrogyria. Or more specifically, cortical malformations associated with 22q11.2DS may surface along a continuum, ranging from severe polymicrogyria, easily diagnosed by radiologists, to more subtle forms of gyral malformations, which require quantitative tools such as $l$ GI.

\section{Effect of CHD}

Similarities in neuroanatomical findings in patients with 22q11.2DS and non-syndromic patients with CHD (i.e. microcephaly, cortical malformations, agenesis of the corpus callosum, open operculum, and white matter lesions ${ }^{10,11}$ ) suggest a strong association between cardiac and cerebral anomalies. In the present report, using stringent criteria, we observed reduced total brain volume in patients with 22q11.2DS with CHD compared with those without CHD. We also found decreased lGI, in the absence of cortical thickness changes, in the parieto-temporo-occipital regions of individuals with 22q11.2DS and CHD. Although our sample sizes were small, the IQ scores of our CHD subgroups suggest an effect of CHD on intellectual functioning in 22q11.2DS. Namely, the verbalnonverbal IQ dissociation during childhood ${ }^{16}$ and lower VIQ in adults ${ }^{29}$ appear to differ between CHD subgroups. If these effects can be replicated in larger samples of patients, the IQ differences would suggest that certain hallmark features of the 22q11.2DS cognitive phenotype might be related to the presence of cardiac malformations.
Two different interpretations can be considered to explain the observed association between brain anomalies and CHD in 22q11.2DS. A first explanation would be that common mechanisms disrupt the development of the cardiovascular and nervous systems, leading to the co-occurrence of both anomalies in one patient. For example, knock-out mice support a role for the TBX1 gene in cardiac defects. ${ }^{30}$ If TBX1 is also expressed in the developing brain, ${ }^{30}$ the extent to which TBX1 may interact with cortical development remains unknown. Alternatively, cardiac malformations may pose a threat to cerebral hemodynamics, a phenomenon that could occur at different points in brain development. First, it has been postulated that CHD prevents 'brain sparing', an in utero protective mechanism that maintains a constant cerebral perfusion in case of reduced placental blood flow. ${ }^{31}$ Second, decreased cerebral blood flow may occur after birth, due to inefficiency in the malformed heart. Finally, surgery itself can be a potential cause of lesions on the developing brain. The fact that most cerebral alterations associated with CHD in non-syndromic patients are apparent at birth points to prenatal injury. ${ }^{10}$ Our ability to identify the precise timing of the alterations, which will be critical for improving treatment, will rely on future studies using fetal or neonatal presurgical imaging. In the meantime, we can speculate that the mechanisms by which cardiac defects impair cortical development before and after birth may be similar. Indeed, almost all of the above-mentioned putative mechanisms result in global hypoperfusion on the growing brain. In the only histopathological report published to date ${ }^{32}$ the younger patients with a history of CHD showed evidence of focal tissue destruction that may have been caused by repeated hypotensive events. Studies of adults with carotid disease have taught us that the most distal fields of the main arterial territories are the regions at highest risk for low cerebral perfusion pressure, resulting in 'watershed' infarcts. ${ }^{33}$ The parieto-temporo-occipital junction corresponds topographically to the juncture of the most distal field of the three main cerebral arteries. The bilateral reduction in $l$ GI observed in affected individuals with CHD could thus be similarly caused by a global hemodynamic failure largely affecting the developing cortex at its most susceptible location. The CHD analyses in the present study support a potential effect of hemodynamic factors on brain development in 22q11.2DS, and illustrate the potential for identifying factors that may contribute to heterogeneity of genetic expression throughout development.

\section{CONCLUSION}

The present study demonstrates how precise delineation of cortical anomalies can shed light on volumetric changes in 
22q11.2DS, and how future work on cortical anomalies may help us to understand both disrupted neurodevelopment and the resulting behavioral and cognitive pheno- type. Further, the current study supports cardiac malformations as a potential determinant of neurodevelopment and prognosis in the syndrome.

\section{REFERENCES}

1. Tezenas Du Montcel S, Mendizabai H, Ayme S, Levy A, Philip N. Prevalence of 22q11 microdeletion. 7 Med Genet 1996; 33: 719 .

2. Simon TJ, Ding L, Bish JP, McDonaldMcGinn DM, Zackai EH, Gee J. Volumetric, connective, and morphologic changes in the brains of children with chromosome 22q11.2 deletion syndrome: an integrative study. Neuroimage 2005; 25: 169-80.

3. Eliez S, Schmitt JE, White CD, Reiss AL. Children and adolescents with velocardiofacial syndrome: a volumetric MRI study. Am $\mathcal{F}$ Psychiatry 2000; 157: 409-15.

4. Kates WR, Burnette CP, Jabs EW, et al. Regional cortical white matter reductions in velocardiofacial syndrome: a volumetric MRI analysis. Biol Psychiatry 2001; 49: 677-84.

5. Gerdes M, Solot C, Wang PP, et al. Cognitive and behavior profile of preschool children with chromosome 22q11.2 deletion. Am f Med Genet 1999; 85: 127-33.

6. Bingham PM, Zimmerman RA, McDonald-McGinn D, Driscoll D, Emanuel BS, Zackai E. Enlarged Sylvian fissures in infants with interstitial deletion of chromosome 22q11. Am f Med Genet 1997; 74: 538-43.

7. Schaer M, Schmitt JE, Glaser B, Lazeyras F, Delavelle J, Eliez S. Abnormal patterns of cortical gyrification in velo-cardio-facial syndrome (deletion 22q11.2): an MRI study. Psychiatry Res 2006; 146: 1-11.

8. Schaer M, Cuadra MB, Tamarit L, Lazeyras F, Eliez S, Thiran JP. A surface-based approach to quantify local cortical gyrification. IEEE Trans Med Imaging 2008; 27 : 161-70.

9. McDonald-McGinn DM, Kirschner R, Goldmuntz E, et al. The Philadelphia story: the 22q11.2 deletion: report on 250 patients. Genet Couns 1999; 10: 11-24.

10. Glauser TA, Rorke LB, Weinberg PM, Clancy RR. Congenital brain anomalies associated with the hypoplastic left heart syndrome. Pediatrics 1990; 85: 984-90.

11. Miller SP, McQuillen PS. Neurology of congenital heart disease: insight from brain imaging. Arch Dis Child Fetal Neonatal Ed 2007; 92: F435-37.

12. Bearden CE, van Erp TG, Dutton RA, et al. Mapping cortical thickness in children with 22q11.2 deletions. Cereb Cortex 2007; 17: 1889-98.

13. Achenbach TM. Manual for the Child Behavior Checklist 4-18 and 1991 Profile. Burlington, VT: University of Vermont, Department of Psychiatry, 1991.

14. Achenbach TM. Manual for the Youth Self-Report Form and Profile. Burlington, VT: University of Vermont, Department of Psychiatry, 1991.

15. Achenbach TM, Rescorla LA. Manual for the ASEBA adults forms and profile. Burlington, VT: University of Vermont, Department of Psychiatry, 2003.

16. Swillen A, Devriendt K, Legius E, et al. The behavioural phenotype in velo-cardio-facial syndrome (VCFS): from infancy to adolescence. Genet Couns 1999; 10: 79 88.

17. Fischl B, Liu A, Dale AM. Automated manifold surgery: constructing geometrically accurate and topologically correct models of the human cerebral cortex. IEEE Trans Med Imaging 2001; 20: 70-80.

18. Zilles K, Armstrong E, Schleicher A, Kretschmann HJ. The human pattern of gyrification in the cerebral cortex. Anat Embryol (Berl) 1988; 179: 173-79.

19. Genovese CR, Lazar NA, Nichols $T$. Thresholding of statistical maps in functional neuroimaging using the false discovery rate. Neuroimage 2002; 15: 870-78.

20. Rakic P. Radial unit hypothesis of neocortical expansion. Novartis Found Symp 2000; 228: 30-42; discussion p 52.

21. Eliez S, Blasey CM, Menon V, White CD, Schmitt JE, Reiss AL. Functional brain imaging study of mathematical reasoning abilities in velocardiofacial syndrome (del22q11.2). Genet Med 2001; 3: 49-55.

22. Barnea-Goraly N, Menon V, Krasnow B, Ko A, Reiss A, Eliez S. Investigation of white matter structure in velocardiofacial syndrome: a diffusion tensor imaging study. Am f Psychiatry 2003; 160: 1863-69.

23. Gothelf D, Presburger G, Levy D, et al. Genetic, developmental, and phys- ical factors associated with attention deficit hyperactivity disorder in patients with velocardiofacial syndrome. $A m \mathcal{f}$ Med Genet B Neuropsychiatr Genet. 2004; 126: 116-21.

24. Dufour F, Schaer M, Debbane M, Farhoumand R, Glaser B, Eliez S. Cingulate gyral reductions are related to low executive functioning and psychotic symptoms in 22q11.2 deletion syndrome. Neuropsychologia 2008; 46: 2986-92.

25. Van Aken K, De Smedt B, Van Roie A, et al. Motor development in schoolaged children with 22q11 deletion (velocardiofacial/DiGeorge syndrome). Dev Med Child Neurol 2007; 49: 210-13.

26. Scambler PJ. The 22q11 deletion syndromes. Hum Mol Genet. 2000; 9: 2421-26.

27. Robin NH, Taylor CJ, McDonald-McGinn DM, et al. Polymicrogyria and deletion $22 \mathrm{q} 11.2$ syndrome: window to the etiology of a common cortical malformation. Am f Med Genet A 2006; 140: 241625 .

28. Barkovich AJ, Rowley H, Bollen A. Correlation of prenatal events with the development of polymicrogyria. A7NR Am 7 Neuroradiol 1995; 16 (Suppl. 4): 822-27.

29. Gothelf D, Penniman L, Gu E, Eliez S, Reiss AL. Developmental trajectories of brain structure in adolescents with 22q11.2 deletion syndrome: a longitudinal study. Schizophr Res 2007; 96: 72-81.

30. Lindsay EA, Vitelli F, Su H, et al. Tbx1 haploinsufficieny in the DiGeorge syndrome region causes aortic arch defects in mice. Nature 2001; 410: 97-101.

31. Donofrio MT, Bremer YA, Schieken RM, et al. Autoregulation of cerebral blood flow in fetuses with congenital heart disease: the brain sparing effect. Pediatr Cardiol 2003; 24: 436-43.

32. Kiehl TR, Chow EW, Mikulis DJ, George SR, Bassett AS. Neuropathologic Features in Adults with 22q11.2 Deletion Syndrome. Cereb Cortex 2009; 19: 153-64.

33. Momjian-Mayor I, Baron JC. The pathophysiology of watershed infarction in internal carotid artery disease: review of cerebral perfusion studies. Stroke 2005; 36: $567-77$. 